Efficient synthesis of spiro[diindenopyridine-indoline]triones catalysed by $\mathrm{PEGOSO}_{3} \mathrm{H}$ $\mathrm{H}_{2} \mathrm{O}$ and [NMP] $\mathrm{H}_{2} \mathrm{PO}_{4}$

Jayant Sindhu $^{\text {a }}$, Harjinder Singh ${ }^{\text {a }}$ and J. M. Khurana ${ }^{* a}$

${ }^{a}$ Department of Chemistry, University of Delhi, Delhi-110007, India.

E-mail: jmkhurana@chemistry.du.ac.in

Procedure for synthesis of compounds 4a-4n using PEG-OSO $3 \mathrm{H}$ in water under conventional heating conditions.

A mixture of indan-1,3-dione $(2.0 \mathrm{mmol})$, substituted isatin $(1.0 \mathrm{mmol})$, aromatic amine $(1.0$ $\mathrm{mmol})$, $\mathrm{PEG}-\mathrm{OSO}_{3} \mathrm{H}(30 \mathrm{~mol} \%, 1.8 \mathrm{~g})$ and water $(10 \mathrm{~mL})$ was placed in a $50 \mathrm{~mL}$ roundbottomed flask. The mixture was heated in an oil bath maintained at $80^{\circ} \mathrm{C}$ for appropriate time as mentioned in Table 2. After completion of reaction as monitored by TLC using ethyl acetate: petroleum ether $(30: 70, \mathrm{v} / \mathrm{v})$ as eluent, the reaction mixture was allowed to cool to room temperature. The precipitate formed was collected by filtration at pump, washed with water, followed by cold ethanol and dried to yield pure 5-aryl-5H-spiro[diindeno[1,2- $b: 2^{\prime}, 1^{\prime}-$ e]pyridine-11,3'-indoline]-2',10,12-triones (4a-4n) in high yields.

Procedure for preparation of compounds $4 \mathrm{a}-4 \mathrm{n}$ using $\mathrm{PEG}^{-O S \mathrm{O}_{3} \mathrm{H}}$ as catalyst in water under ultrasonic irradiation.

A mixture of indan-1,3-dione $(0.96 \mathrm{mmol})$, substituted isatin $(0.48 \mathrm{mmol})$, aromatic amine (0.48), $\mathrm{PEG}-\mathrm{OSO}_{3} \mathrm{H}(30 \mathrm{~mol} \%, 0.84 \mathrm{~g})$ and water $(5 \mathrm{~mL})$ was placed in a $50 \mathrm{~mL}$ roundbottomed flask. The mixture was sonicated at $40^{\circ} \mathrm{C}$ for appropriate time as mentioned in Table 2. After completion of reaction as monitored by TLC as above, the reaction mixture was allowed to cool to room temperature. The precipitate formed was collected by filtration 
at pump, washed first with water, then with cold ethanol and dried to yield pure products $\mathbf{4 a -}$ 4n in high yields.

Procedure for preparation of compounds $4 a-4 n$ using $\left[\mathrm{NMP}_{3} \mathrm{H}_{2} \mathrm{PO}_{4}\right.$ as catalyst under conventional heating conditions.

A mixture of indan-1,3-dione $(0.96 \mathrm{mmol})$, substituted isatin $(0.48 \mathrm{mmol})$, aromatic amine $(0.48 \mathrm{mmol})$ and $[\mathrm{NMP}] \mathrm{H}_{2} \mathrm{PO}_{4}(30 \mathrm{~mol} \%)$ was placed in a $50 \mathrm{~mL}$ round-bottomed flask. The mixture was heated in an oil bath maintained at $80^{\circ} \mathrm{C}$ for appropriate time as mentioned in Table 3. After completion of reaction as monitored by TLC using ethyl acetate: petroleum ether $(30: 70, \mathrm{v} / \mathrm{v})$ as eluent, the reaction mixture was worked up as above to yield pure 5-aryl$5 H$-spiro[diindeno[1,2-b:2',1'-e]pyridine-11,3'-indoline]-2',10,12-triones $\mathbf{( 4 a - 4 n ) ~ i n ~ h i g h ~}$ yields.

Procedure for preparation of compounds $4 a-4 n$ using $[\mathrm{NMP}] \mathrm{H}_{2} \mathrm{PO}_{4}$ as catalyst under ultrasonic irradiation.

A mixture of indan-1,3-dione $(0.96 \mathrm{mmol})$, substituted isatin $(0.48 \mathrm{mmol})$, aromatic amine $(0.48 \mathrm{mmol})$ and $[\mathrm{NMP}] \mathrm{H}_{2} \mathrm{PO}_{4}(30 \mathrm{~mol} \%)$ was placed in a $50 \mathrm{~mL}$ round-bottomed flask. The mixture was sonicated at $40^{\circ} \mathrm{C}$ for appropriate time as mentioned in Table 3. After completion of reaction, the products $\mathbf{4 a - 4 n}$ were collected after filtration at pump, washing with water, followed by cold ethanol and drying. Pure 5-aryl-5H-spiro[diindeno[1,2- $b: 2$ ', 1 'e]pyridine-11,3'-indoline]-2',10,12-triones (4a-4n) were obtained in high yields.

\section{Spectral data}

5-(4-Methoxyphenyl)-5H-spiro[diindeno[1,2-b:2',1'-e]pyridine-11,3'-indoline]-2',10,12trione (4a) ${ }^{14} \mathrm{mp}>300{ }^{\circ} \mathrm{C}$ (ethanol); ${ }^{1} \mathrm{H}$ NMR $\left(400 \mathrm{MHz}, \mathrm{DMSO}, d_{6}\right) \delta_{\mathrm{H}}: 10.62(1 \mathrm{H}, \mathrm{s}, \mathrm{NH})$, 8.04-8.01 (1H, m, Ar-H), 7.83-7.80 (1H, m, Ar-H), 7.38-7.36 (1H, m, Ar-H), 7.30-7.23 (6H, $\mathrm{m}, \mathrm{Ar}-\mathrm{H}), 7.19-7.14(3 \mathrm{H}, \mathrm{m}, \mathrm{Ar}-\mathrm{H}), 6.89-6.84(2 \mathrm{H}, \mathrm{m}, \mathrm{Ar}-\mathrm{H}), 5.61\left(2 \mathrm{H}, \mathrm{d},{ }^{3} J_{\mathrm{HH}}=7.2 \mathrm{~Hz}\right.$, ArH), $3.95\left(3 \mathrm{H}, \mathrm{s}, \mathrm{OCH}_{3}\right) ;{ }^{13} \mathrm{C} \mathrm{NMR}\left(100 \mathrm{MHz}, \mathrm{DMSO}, d_{6}\right) \delta_{\mathrm{C}}: 189.7,177.7,161.0,156.3$, 
$142.1,136.2,134.4,132.5,132.4,131.1,130.9,130.3,128.6,124.5,121.6,121.5,115.3$, 115.2, 113.4, 111.4, 109.1, 55.8.

5-(4-Methylphenyl)-5H-spiro[diindeno[1,2-b:2',1'-e]pyridine-11,3'-indoline]-2',10,12-

trione (4b) ${ }^{14} \mathrm{mp}>300{ }^{\circ} \mathrm{C}$ (ethanol); ${ }^{1} \mathrm{H}$ NMR $\left(400 \mathrm{MHz}, \mathrm{CDCl}_{3}\right) \delta_{\mathrm{H}}: 7.60(2 \mathrm{H}, \mathrm{m}, \mathrm{H}-\mathrm{Ar})$, 7.47-7.45 (2H, m, H-Ar), 7.30-7.28 (2H, m, H-Ar), 7.22-7.10 (4H, m, H-Ar), 6.94-6.91 (4H, m, H-Ar), $5.55\left(2 \mathrm{H}, \mathrm{d},{ }^{3} \mathrm{~J}_{\mathrm{HH}}=7.2 \mathrm{~Hz}, \mathrm{ArH}\right), 2.61\left(3 \mathrm{H}, \mathrm{s}, \mathrm{CH}_{3}\right) ;{ }^{13} \mathrm{C} \mathrm{NMR}(100 \mathrm{MHz}, \mathrm{DMSO}$, $\left.d_{6}\right) \delta_{\mathrm{C}}: 189.6,177.6,156.0,142.1,141.6,136.2,135.3,134.4,132.5,132.4,130.8,130.7$, $130.3,129.7,128.6,124.5,121.6,121.5,111.4,109.1,30.7$.

5-(4-Nitrophenyl)-5H-spiro[diindeno[1,2-b:2',1'-e]pyridine-11,3'-indoline]-2',10,12trione (4c) ${ }^{14} \mathrm{mp}>300{ }^{\circ} \mathrm{C}$ (ethanol); ${ }^{1} \mathrm{H}$ NMR (400 MHz, DMSO, $\left.d_{6}\right) \delta_{\mathrm{H}}: 10.66(1 \mathrm{H}, \mathrm{s}, \mathrm{NH})$, 8.60-6.87 (14H, m, ArH), $5.55\left(2 \mathrm{H}, \mathrm{d},{ }^{3} J_{\mathrm{HH}}=7.2 \mathrm{~Hz}, \mathrm{ArH}\right)$.

5-(4-Bromophenyl)-5H-spiro[diindeno[1,2-b:2',1'-e]pyridine-11,3'-indoline]-2',10,12trione (4d) ${ }^{14} \mathrm{mp}>300{ }^{\circ} \mathrm{C}$ (ethanol); ${ }^{1} \mathrm{H}$ NMR (400 MHz, DMSO, $\left.d_{6}\right) \delta_{\mathrm{H}}: 10.65(1 \mathrm{H}, \mathrm{s}, \mathrm{NH})$, 8.13-8.15 (1H, m, Ar-H), 8.00-7.93 (3H, m, Ar-H), 7.38-7.36 (1H, m, Ar-H), 7.27-7.22 (4H, m, Ar-H), 7.18-7.13 (3H, m, Ar-H), 6.87-6.82 (2H, m, Ar-H), $5.54\left(2 \mathrm{H}, \mathrm{d},{ }^{3} J_{\mathrm{HH}}=7.2 \mathrm{~Hz}\right.$, $\mathrm{ArH})$.

5-Phenyl-5H-spiro[diindeno[1,2-b:2',1'-e]pyridine-11,3'-indoline]-2',10,12-trione (4e) ${ }^{14}$ $\mathrm{mp}>300{ }^{\circ} \mathrm{C}$ (ethanol); ${ }^{1} \mathrm{H}$ NMR $\left(400 \mathrm{MHz}, \mathrm{DMSO}, d_{6}\right) \delta_{\mathrm{H}}: 10.65(1 \mathrm{H}, \mathrm{s}, \mathrm{NH}), 8.13-8.11$ (1H, m, Ar-H), 7.92-7.84 (2H, m, Ar-H), 7.78-7.74 (2H, m, Ar-H), 7.37-7.35 (1H, m, Ar-H), 7.23-7.22 (4H, m, Ar-H), 7.17-7.13 (1H, m, Ar-H), 7.01-7.06 (2H, m, Ar-H), 6.88-6.82 (2H, $\mathrm{m}, \operatorname{Ar}-\mathrm{H}), 5.43\left(2 \mathrm{H}, \mathrm{d},{ }^{3} J_{\mathrm{HH}}=7.2 \mathrm{~Hz}, \mathrm{ArH}\right) .{ }^{13} \mathrm{C}$ NMR $\left(100 \mathrm{MHz}, \mathrm{DMSO}, d_{6}\right) \delta_{\mathrm{c}}: 189.6$, $177.6,155.8,142.0,138.0,135.8,134.5,132.6,131.8,130.4,128.6,124.2,121.4,111.4$, 109.1, 45.6.

5-(4-Methoxyphenyl)-5'-nitro-5H-spiro[diindeno[1,2-b:2',1'-e]pyridine-11,3'-indoline]2',10,12-trione (4f) ${ }^{\mathbf{1 4}} \mathrm{mp}>300{ }^{\circ} \mathrm{C}$ (ethanol); ${ }^{1} \mathrm{H}$ NMR (400 MHz, DMSO, $\left.d_{6}\right) \delta_{\mathrm{H}}: 11.3(1 \mathrm{H}$, 
s, NH), 8.40 (2H, m, Ar-H), 8.18-8.10 (2H, m, Ar-H), 7.81-7.78 (1H, m, Ar-H), 7.30-7.06 $(8 \mathrm{H}, \mathrm{m}, \mathrm{Ar}-\mathrm{H}) 5.59\left(2 \mathrm{H}, \mathrm{d},{ }^{3} J_{\mathrm{HH}}=7.6 \mathrm{~Hz}, \mathrm{ArH}\right), 3.93\left(3 \mathrm{H}, \mathrm{s}, \mathrm{OCH}_{3}\right) ;{ }^{13} \mathrm{C} \mathrm{NMR}(100 \mathrm{MHz}$, DMSO, $\left.d_{6}\right) \delta_{\mathrm{C}}: 189.7,178.4,161.1,157.2,148.8,142.6,136.2,135.0,132.7,132.3,131.3$, $130.8,130.6,130.2,126.2,121.9,121.6,120.5,115.3,115.1,110.1,109.3,55.8$.

5'-Nitro-5-(4-nitrophenyl)-5H-spiro[diindeno[1,2-b:2',1'-e]pyridine-11,3'-indoline]2',10,12-trione (4g) ${ }^{\mathbf{1 4}} \mathrm{mp}>300{ }^{\circ} \mathrm{C}$ (ethanol); ${ }^{1} \mathrm{H}$ NMR (400 MHz, DMSO, $\left.d_{6}\right) \delta_{\mathrm{H}}: 11.43(1 \mathrm{H}$, s, NH), 7.09-8.67 (13H, m, ArH), $5.58\left(2 \mathrm{H}, \mathrm{d},{ }^{3} J_{\mathrm{HH}}=7.5 \mathrm{~Hz}, \mathrm{ArH}\right) ;{ }^{13} \mathrm{C} \mathrm{NMR}(100 \mathrm{MHz}$, DMSO, $\left.d_{6}\right) \delta_{\mathrm{C}}: 189.7,178.4,156.6,149.7,149.2,143.4,143.0,136.2,135.3,133.4132 .4$, $131.0,126.7,126.0,122.4,122.1,121.1,110.8,109.7$.

5'-Nitro-5-(4-methylphenyl)-5H-spiro[diindeno[1,2-b:2',1'-e]pyridine-11,3'-indoline]2',10,12-trione (4h) ${ }^{\mathbf{1 4}} \mathrm{mp}>300{ }^{\circ} \mathrm{C}$ (ethanol); ${ }^{1} \mathrm{H}$ NMR (400 MHz, DMSO, $\left.d_{6}\right) \delta_{\mathrm{H}}: 11.38(1 \mathrm{H}$, s, NH), 8.41-8.40 (1H, m, ArH), 8.18-8.15 (1H, m, ArH), 8.10-8.08 (1H, m, ArH), 7.78-7.75 $(1 \mathrm{H}, \mathrm{m}, \mathrm{ArH}), 7.59-7.23(4 \mathrm{H}, \mathrm{m}, \mathrm{ArH}), 7.14-7.06(3 \mathrm{H}, \mathrm{m}, \mathrm{ArH}), 5.52\left(2 \mathrm{H}, \mathrm{d},{ }^{3} \mathrm{~J}_{\mathrm{HH}}=7.6 \mathrm{~Hz}\right.$, ArH), $2.54\left(3 \mathrm{H}, \mathrm{s}, \mathrm{CH}_{3}\right) ;{ }^{13} \mathrm{C}$ NMR $\left(100 \mathrm{MHz}, \mathrm{DMSO}, d_{6}\right) \delta_{\mathrm{C}}: 189.7,178.4,156.8,148.8$, $142.6,141.6,136.1,135.2,135.0,132.6,132.3,130.7,130.6,129.9,129.3,126.2,121.8$, 121.6, 120.5, 110.1, 109.2, 30.7 .

5-(4-Bromophenyl)-5'-nitro-5H-spiro[diindeno[1,2-b:2',1'-e]pyridine-11,3'-indoline]2',10,12-trione (4i) ${ }^{\mathbf{1 4}} \mathrm{mp}>300{ }^{\circ} \mathrm{C}$ (ethanol); ${ }^{1} \mathrm{H}$ NMR (400 MHz, DMSO, $\left.d_{6}\right) \delta_{\mathrm{H}}: 11.42(1 \mathrm{H}$, s, NH), 8.46-7.09 (13H, m, ArH), $5.57\left(2 \mathrm{H}, \mathrm{d},{ }^{3} J_{\mathrm{HH}}=6.0 \mathrm{~Hz}, \mathrm{ArH}\right)$.

5'-Nitro-5-phenyl-5H-spiro[diindeno[1,2-b:2',1'-e]pyridine-11,3'-indoline]-2',10,12trione (4j) ${ }^{\mathbf{1 4}} \mathrm{mp}>300{ }^{\circ} \mathrm{C}$ (ethanol); ${ }^{1} \mathrm{H}$ NMR (400 MHz, DMSO, $\left.d_{6}\right) \delta_{\mathrm{H}}: 11.41(1 \mathrm{H}, \mathrm{s}, \mathrm{NH})$, 8.45-7.10(14H, m, ArH), $5.48\left(2 \mathrm{H}, \mathrm{d},{ }^{3} J_{\mathrm{HH}}=7.2 \mathrm{~Hz}, \mathrm{ArH}\right)$.

5'-Bromo-5-(4-methylphenyl)-5H-spiro[diindeno[1,2-b:2',1'-e]pyridine-11,3'-indoline]2',10,12-trione (4k) ${ }^{\mathbf{1 4}} \mathrm{mp}>300{ }^{\circ} \mathrm{C}$ (ethanol); ${ }^{1} \mathrm{H}$ NMR (400 MHz, DMSO, $\left.d_{6}\right) \delta_{\mathrm{H}}: 10.7(1 \mathrm{H}, \mathrm{s}$, NH), 8.04-8.01 (1H, m, ArH), 7.74-7.29 (1H, m, ArH), 7.63-7.51(3H, m, ArH), 7.33- 
7.31(1H, m, ArH), 7.23-7.21(4H, m ArH), 7.11-7.07(2H, m, ArH), 6.82-6.70(1H, m, ArH) 5.49-5.47(2H, d, $\left.{ }^{3} J_{\mathrm{HH}}=8, \mathrm{ArH}\right), 2.52\left(3 \mathrm{H}, \mathrm{s}, \mathrm{CH}_{3}\right) ;{ }^{13} \mathrm{C} \mathrm{NMR}\left(100 \mathrm{MHz}, \mathrm{DMSO}, d_{6}\right) \delta_{\mathrm{C}}$ : $189.7,177.5,156.5,141.6,136.6,136.2,135.3,132.5,132.4,131.4,130.7,130.5,129.9$, $129.4,127.4,121.6,113.6,111.1,110.7,46.0,21.2$.

5'-Bromo-5-phenyl-5H-spiro[diindeno[1,2-b:2',1'-e]pyridine-11,3'-indoline]-2',10,12trione (4l) ${ }^{14} \mathrm{mp}>300^{\circ} \mathrm{C}$ (ethanol); ${ }^{1} \mathrm{H}$ NMR (400 MHz, DMSO, $\left.d_{6}\right) \delta_{\mathrm{H}}: 10.7(1 \mathrm{H}, \mathrm{s}, \mathrm{NH})$, 8.20-8.18 (1H, m, ArH), 7.89-7.67 (5H, m, ArH), 7.34-7.32(1H, m, ArH), 7.24-7.23(4H, m, ArH), 7.11-7.08(2H, m ArH), 6.81-6.79(1H, m, ArH) 5.42-5.40(2H, d, $\left.{ }^{3} J_{\mathrm{HH}}=7.2, \mathrm{ArH}\right) ;{ }^{13} \mathrm{C}$ NMR $\left(100 \mathrm{MHz}, \mathrm{DMSO}, d_{6}\right) \delta_{\mathrm{C}}: 189.6,177.3,156.2,141.5,137.8,136.5,136.1,132.5$, $132.3,131.7,131.3,130.5,130.3,127.3,121.6,121.5,113.5,111.0,110.7,45.9$.

\section{5'-Bromo-5-(4-nitrophenyl)-5H-spiro[diindeno[1,2-b:2',1'-e]pyridine-11,3'-indoline]-}

2',10,12-trione (4m) ${ }^{\mathbf{1 4}} \mathrm{mp}>300^{\circ} \mathrm{C}$ (ethanol); ${ }^{1} \mathrm{H}$ NMR (400 MHz, DMSO, $\left.d_{6}\right) \delta_{\mathrm{H}}: 10.7(1 \mathrm{H}$, s, NH), 8.61-8.54 (3H, m, ArH), 8.27-8.14 (1H, m, ArH), 7.71(1H, m, ArH), 7.35-7.24(5H, m ArH), 7.12-7.08(2H, m, ArH) 6.82-6.70(1H, m, ArH), 5.53-5.51(2H, d, $\left.{ }^{3} J_{\mathrm{HH}}=7.2, \mathrm{ArH}\right)$.

5'-Bromo-5-(4-methoxyphenyl)-5H-spiro[diindeno[1,2-b:2',1'-e ]pyridine-11,3'-indoline]2',10,12-trione (4n) ${ }^{\mathbf{1 4}} \mathrm{mp}>300{ }^{\circ} \mathrm{C}$ (ethanol); ${ }^{1} \mathrm{H}$ NMR (400 MHz, DMSO, $\left.d_{6}\right) \delta_{\mathrm{H}}: 10.7(1 \mathrm{H}, \mathrm{s}$, $\mathrm{NH})$, 8.08-8.06 (1H, m, ArH), 7.78-7.65(1H, m, ArH), 7.33-7.13(10H, m ArH), 6.81$6.79(1 \mathrm{H}, \mathrm{m}, \mathrm{ArH}), 5.56-5.55\left(2 \mathrm{H}, \mathrm{d},{ }^{3} \mathrm{~J}_{\mathrm{HH}}=6.8, \mathrm{ArH}\right), 3.92(3 \mathrm{H}, \mathrm{s}, \mathrm{OMe}) ;{ }^{13} \mathrm{C} \mathrm{NMR}(100$ MHz, DMSO, $\left.d_{6}\right) \delta_{\mathrm{C}}: 189.6,177.3,161.0,156.6,141.5,136.5,136.1,132.5,132.3,131.2$, $130.8,130.4,130.2,121.6,121.5,115.2,115.0,113.4,111.0,110.7,55.8,45.9$. 